Vol. 4, Special Issue 2, January 2017

\title{
Red Signal Alerting for Train using Wireless Communication
}

\author{
Mr. Sagar Shejval ${ }^{1}$, Mr. Dodake R.R ${ }^{2}$ \\ Student -Bachelor of Engineering, Department of Electronics \& Telecommunication Engineering, Dr. Daulatrao Aher \\ College of Engineering, Karad, M.S. India ${ }^{1}$ \\ Assistant Professor, Department of Electronics \& Telecommunication Engineering, Dr. Daulatrao Aher College of \\ Engineering, Karad , M.S. India ${ }^{2}$
}

\begin{abstract}
Human negligence and human error have becomes the primary reason for many train accident in India. The primary goal of this project is to avoid train accident and train collision using wireless communication. Our system is useful for train driver. To maintain the train flow, massive amounts of signals are required at pre-set distances. Traditionally, a train driver has to keep a constant lookout for any red signals that might appear on the post. The train driver then decides whether to stop or to continue with his designated path on the basis of these signals. But it's quite troublesome for the drivers to keep an eye out for every visual sign. To make life for train drivers and the maintenance staff as well easier, engineers have come up with an ingenious technology named as wireless red signal alerting for trains. At this time train will be gradually slow they also make for a good control systems.
\end{abstract}

Keywords: RF Module, Microcontroller, REED Switch, Embedded System.

\section{INTRODUCTION}

The Indian Railway network is one of the biggest rail networks in the world. Indian railway Network Handling and managing such a vast network is not an easy. The rail network consists of a many junctions and many signals set distances to manage the train flow. So far that train driver needs to linearly check for any red signals on every post and decide whether train stop or move train on station. It is very difficult to linearly keep track of every individual signal for the drive.

The propose an automatic alerting system that alerts the driver of any red signal ahead. Full assembling works on the basis of RF technology. A timer circuit is used in the transmitter circuit and this transmitter circuit is placed at a signal pole.

Transmitter circuit to produced RF beam between the pulses for a particular time interval. Then switch is turned ON only when the RED signal is ON. These RF beam cycles are repeated until the RED signal is ON. The transmitter linearly transmits RF signals informing about RED signal.

An $\mathrm{RF}$ receiver circuit is placed in the train. This is placed in such a way that it receives RF beam. Then the train whenever comes between the certain range area of the RF transmitter, $\mathrm{RF}$ receiver circuit receives the $\mathrm{RF}$ signal as input and sends it to the microcontroller.

The microcontroller then processes this receiving data for alert the driver about the signal ahead of train.

\section{PROPOSED SYSTEM}

The goal of this work is to design and implement automatic red signal alerting in railway system to prevent the train accident. In this project transmitter fitted on signal pole, then RED signal is turned ON through control room. Transmitter continuous transmits RF waves informing the signal. The receiver circuit fitted in train. Trains come in proper range of the RF transmitter. Capture this signal and demodulate this signal. Microcontroller process on it then alert the driver and automatically motor will be slow.

\section{BLOCK DIAGRAM:}

Transmitter:

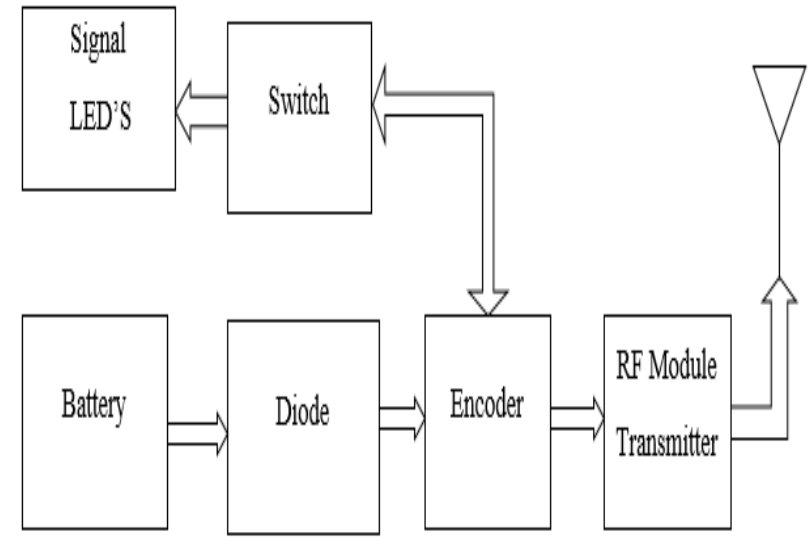

Receiver: 


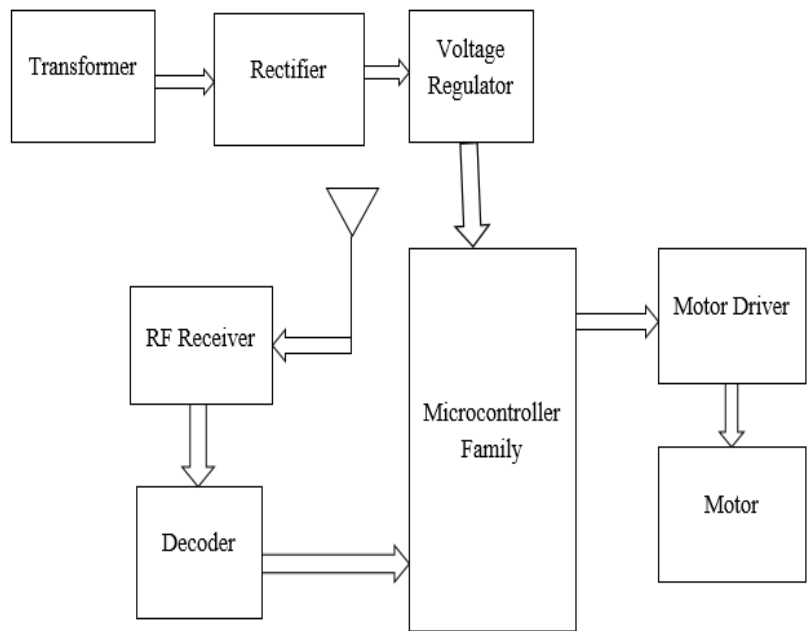

Fig.1 Block diagram of proposed system

\section{WORKING}

First of all whenever any train present on railway station. Transmitter circuit fitted on signal pole .red signal turn ON through control room. Now our system get started, Encoder are used analog signal is converted into digital signal and this signal continuously transmittedby RFtransmitter. Here whenever any train comes in the range of $2 \mathrm{~km}$ at that time, receiver module already fitted in train. It will be receive RF signal and demodulate this signal.i.e.analog signal. This analog signal goes to microcontroller will be process on it, then automatically motor will be gradually slow this process will be controlled motor driver IC.

\section{HARDWARE REQUIREMENTS}

4.1 Microcontroller.

4.2 Power supply.

4.3 RF Module.

4.4 Motor Driver IC.

4.5 Voltage Regulator.

\subsection{MICROCONTROLLER:}

The AT89S51 is useful as low-power, It is highperformance CMOS 8-bit microcontroller within 4Kbytes of in-system programmable Flash memory. The device have a high-density nonvolatile memory technology and is compatible with the industry-standard.Microcontroller 80C51 including all instruction set and pin out as general microcontroller.The Atmel AT89S51 is a powerful microcontroller which provides a highly-flexible ancosteffective solution to many embedded control applications.

Features of AT89S51:

○ The AT89S51 is 4K bytes of Flash.

- 128 bytes of on chip data memory.(RAM)

- 32 I/O lines.

- Four register bank.

- Two data pointers.
○ Two 16-bit timer/counters,

- Five vector two-level interrupt architecture.

- A full duplex serial port.

o On chip oscillator and clock circuitry.

In addition, the AT89S51 is work with static logic for operation down to zero frequency and supports two software selectable power saving modes.

The Idle Mode they will stops the CPU. while allowing the RAM, timer/counters, serial port, and Interrupt system to continue functioning. They will disabling all other chip functions until the next external interrupt or hardware reset.

\subsection{POWER SUPPLY:}

Any electronic circuit required a power supply. It will consist of transformer, rectifier, and regulator. Main function of power supply is to convert one form of electric energy to another form as a result, this power supply referred to electric power converters. Power supply is used to provide the power to various circuits in the system. The power supply is to be properly designed so that current requirement each block in the system fulfilled. The power supply unit is converts main AC to low voltage DC power.

\subsection{RF MODULE:}

RF Module is the heart of wireless communication. Generally, the wireless systems designer has two overriding constraints: Radio frequency operate over a certain amount of distance and transfer a certain amount of information within a data rate. The RF modules are small in dimension and have a wide in operating voltage range i.e. $3 \mathrm{~V}$ to $12 \mathrm{~V}$.

The RF module name suggests, operates at Radio Frequency. The corresponding range of Radio frequency varies between $30 \mathrm{kHz}$ to $300 \mathrm{GHz}$. In this RF system, the digital data is represented as difference in the amplitude of carrier wave. This modulation is called as Amplitude Shift Keying (ASK).

$\mathrm{RF}$ transmission is more strong and reliable than IR transmission. Radio frequency communication uses a specific frequency. The performance of an RF module will depends on several factors such that increasing the transmitter's power causes a large communication distance will be gathered. However it has consume high electrical power drain on the transmitter device, it results shorter operating life of the battery for powered devices. Also it has an chances of higher transmitted power get create interference with other RF devices.

\subsection{MOTOR DRIVER IC:}

Motor Driver ICL293D which allows DC motor to drive on either direction. Motor driver IC has a 16-pin IC. which can control a set of two DC motors simultaneously in any direction. It means that you can control two DC motor with a single L293D IC. Dual H-bridge Motor Driver integrated circuit(IC).The L293D can alsodrive small and quiet big motors. 


\subsection{VOLTAGE REGULATOR:}

The voltage regulator can be used for the voltage regulation purpose. It use IC 7805 voltage regulator. The IC number has a specific significance. The number 78 represents the series number while 05 represent the output voltage generated by the IC.Voltage regulator is used for regulate the voltage.

\section{SOFTWARE REQUIREMENTS}

The Keil C51 C Compiler is the most popular $8051 \mathrm{C}$ compiler in the world. It supply's more features than any other C compiler. It give you allows to write 8051 microcontroller applications in $\mathrm{C}$ that have the efficiency and speed of assembly language. Also it give you full access to all resources of the 8051. The C51 Compiler translates $\mathrm{C}$ source files into re-locatable object modules which contain full symbolic information for debugging with the $\mu$ Vision Debugger or an in-circuit emulator. In add any other object file, the compiler generates a listing file which include symbol table and cross reference information.

\section{CONCLUSION}

Study of implementing wireless red signal alerting for trains. This is innovative system design to avoid any train accident. In Indian railways train accident happening by doesn't managing the railway timetable so far we suggestion a stop the train automatically. This technology improves the transportation through the railway system and makes it much safer. We believe that success depends on both the railway industry and the regulator working together to achieve that common goal.

\section{APPLICATIONS AND FUTURE SCOPE}

- Manage timetable of railways

- Give more flexibility.

- It can avoid train accidents caused by the extreme weather conditions.

- More helpful for trackman.

By using this system can be developed modern signal alerting system. Also by this technology can be directly stop train automatically from main control room.

\section{REFERENCES}

[1] Mr. Pawan Kumar, Mr. Kiran J Kumbhar, "Implementation of train accident Avoidance using RF Technology", International Journal Of Research in Advent Technology, May2015.

[2] Kiruthiga M., Dhivya M.M., "Wireless Communication System for Railway Signal Automation at Unmanned Level",International conference of engineering technology and science, Feb 2014

[3] Jitendra Grover, "Wireless Sensor Network In Railway Signaling System", April $20155^{\text {th }}$ International conference on communication system And network technology.
[4] J. Banuchandar, V. Kaliraj,P. Balasubramanian, S. Deepa, N.Thamilarasi "automated unmanned railway level crossing system" International Journal of Modern Engineering Research (IJMER) Vol.2, Issue.1, Jan-Feb 2012

[5] K. Vidyasagar, P. Sekhar Babu, R. RamPrasad, "Anti Collision and Secured Level Crossing System". International Journal of Computer Applications (0975 - 8887) December 2014.

[6] Bhatt, Ajaykumar A, "An Anti-Collision Device (ACD) Network A train Collision Prevention System (TCPS) and concept for reducing railway accidents", February, 2005. 